\title{
Importance of potency in typing by colicine production
}

\author{
J. McGEACHIE AND W. McCORMICK \\ From the University Department of Bacteriology, Glasgow Royal Infirmary
}

SYNOPSIS Six colicinogenic strains, producing colicine $A, B, D, K, S_{4}$, and V, showed a variation in the reproducibility of the potency of their crude extracts, although a standard technique was used. The importance of this variation on typing was shown by the effect of different colicine potencies on the patterns of inhibition of the Abbott and Shannon indicators. Using a diffusion technique, 3,004 assays were performed and it was found that the patterns of inhibition depended mainly on the strength of the colicine.

A further source of error in typing by production patterns was the sensitivity of the indicator strain. Two sets of indicators from different sources were compared, and though there was agreement in most strains, there were differences which varied from slightly more resistant to totally resistant.

It is suggested that the potency of the colicine produced by the colicinogenic strain should be known before typing is performed in order that standard comparisons may be achieved. The precise sensitivity of the indicators should also be confirmed periodically.

The interest in colicine production typing by using multiple indicators has been increasing over recent years, and has been applied to the Shigella sonnei and flexneri (Abbott and Shannon, 1958; Gillies, 1964; Hart, 1965); Escherichia coli (Shannon, 1957; Hamon, 1959; McGeachie, 1965); Proteus species (Cradock-Watson, 1965); Klebsiella species (Linton, 1960); and Pseudomonas pyocyanea (Darrell and Wahba, 1964; Wahba, 1965; Osman, 1965; Gillies and Govan, 1966). In most cases the streak method, originally described by Fredericq, Thibault, and Gratia (1946) and subsequently modified by several investigators (Abbott and Shannon, 1958; Gillies, 1964), has been used. We had previously described an agar extraction method (McGeachie and McCormick, 1963; McGeachie, 1965) for classifying the patterns of $E$. coli isolated from the urine, and had noted that several colicines, derived from known colicinogenic strains, had occasionally shown a loss of inhibition of some of the indicators. This prompted us to consider whether the concentration of colicine was the variable feature.

\section{MATERIALS}

COLICINOGENIC STRAINS E. freundii, strain CA 31 producing colicine $\mathrm{A} ; E$. coli, strain $\mathrm{CA} 18$ producing coli-

Received for publication 20 April 1967. cine B; $E$. coli, strain CA 23 producing colicine D; $\boldsymbol{S h}$. dispar, strain $\mathbf{P} 15$ producing colicine $S 4$. These strains were received from Professor P. Fredericq.

$E$. coli, strain CL 131 (K235 of Fredericq) producing colicine $\mathrm{K}$ and $E$. coli, strain CL 1 (CA7 of Fredericq) producing colicine $\mathrm{V}$ were received from Dr. B. A. D. Stocker.

INDICATOR STRAINS Shigella sonnei, strains, 2, 56, 17, 2M, 38, 56/56, 56/98, R6, R1, 2/15, 2/7, 2/64, R5; Shigella dysenteriae, M19 (NCTC 8218); and E. coli, strain Row derived from $E$. coli K12. These indicators are those of Abbott and Shannon. Our stock cultures (referred to as ' $s$ ' in the tables) were obtained from Dr. R. R. Gillies in 1960 and have been stored in the lyophilised state since, subculturing on Dorset's egg medium from time to time. For comparison of sensitivity, a more recent series of these indicators was obtained from Mrs. J. Goodban at Dysentery Reference Laboratories, Colindale; these are referred to as ' $c$ ' in the tables.

$E$. coli, strain ( $\phi$ of Gratia) from Dr.W.F. Goebel.

MEDIA Digest nutrient agar was prepared by adding $1.2 \%$ New Zealand agar (Oxoid) to digest nutrient broth (Cruickshank, 1960).

Peptone water agar for the indicator plates was made as described by Cruickshank (1960). For colicine sensitivity testing equal volumes of a peptone water culture of the indicator organism and $1 \%$ peptone water agar were mixed to give a concentration of $0.5 \%$ seeded agar and poured on the surface of a $1 \%$ peptone water agar base. 
METHODS

AGAR EXTRACTION METHOD This has been described in detail elsewhere (McGeachie, 1965) and consists of a freeze-thaw method to obtain the colicine extracts from the agar.

METHOD FOR TESTING THE TITRE OF COLICINE Bases of $10 \mathrm{ml}$. $1 \%$ peptone water agar were covered with $5 \mathrm{ml}$. seeded $0.5 \%$ peptone water agar. This was seeded with $E$. coli strain phi to give a concentration of approximately $5 \times 10^{7}$ organisms per millilitre agar. The colicine extract was diluted in distilled water and one loopful of the dilution was placed on a plate, similar to the ring method of Goebel, Barry, and Shedlovsky (1956). The end point was the last dilution which produced complete inhibition after 18 hours' incubation; if partial inhibition was present at the next spot this titre was also noted, for example, 64 (128) where complete inhibition occurred at the $1: 64$ dilution and partial $1: 128$. Thus there were between 64 and 128 units in the sample (Fig. 1). Further intermediate dilutions were made to confirm the exact titre.

Several other methods of titre estimation were tested, using a dropper in place of a loop. There was frequently an unsatisfactory result due to the spread of the drop

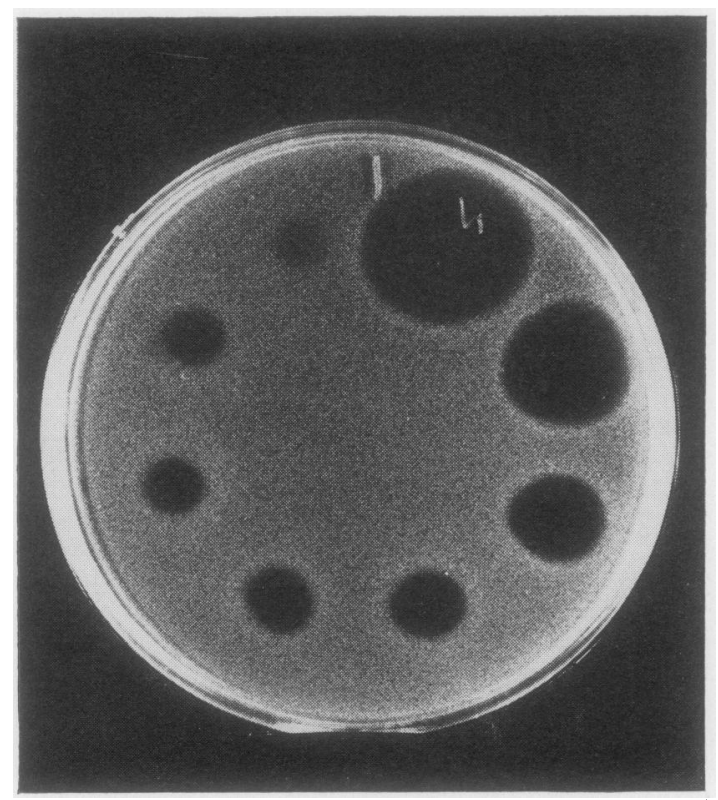

FIG.1. Titre of colicine. The indicator plate has been inoculated with $\mathrm{E}$. coli phi, $5 \times 10^{7}$ cells per ml. peptone water agar. The colicine has been prepared in doubling dilutions from neat to 1 : 512, and a loopful of each dilution has been placed on the surface of the plate. The last zone to show clear inhibition is taken as the end point, in this case the titre is between the seventh and eighth loopful, i. e., $64 / 128$. on the surface of the plate; to overcome this, the drop was contained in a stainless steel cylinder, similar to the Heatley cup. However the end point by the drop method $\overline{0}$ was identical to that obtained by the loop method.

METHOD FOR TESTING THE INHIBITION INDICATORS BY $\frac{\rho}{\partial}$ PUNCH HOLE This method was essentially that previ- 2 ously described (McGeachie and McCormick, 1963; ڤ్ల McGeachie, 1965), where plates, seeded with a concen- $\overrightarrow{-}$ tration similar to that used for the titre plate, of the ? various indicator strains, had holes bored with a number $\overrightarrow{\vec{\omega}}$ 2 cork borer giving a hole of $5 \mathrm{~mm}$. diameter. These holes were filled level to the surface with the colicine extract. The extracts were added in various dilutions, in the main between 1 unit and 1,000 units. Several of the $O$ preparations had a high titre, for example, colicine $\mathrm{K}$ was obtained in a purified preparation of 131,072 units and $\infty$ colicine $A$ in 8,000 units, and these strengths were tested.

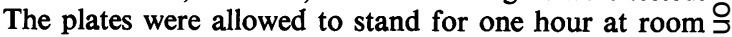
temperature and then incubated at $37^{\circ} \mathrm{C}$. The zone of $\rightarrow$ inhibition was taken as the mean diameter within which $Z$ no growth occurred.

PARTIAL PURIFICATION OF COLICINE EXTRACTS In order $\frac{3}{8}$ to obtain high titre extracts, purification and concentration was performed. The purification of the colicine will $\vec{\varphi}$ be described in detail at a later date. Briefly, the following method was used. The extract was reduced in volume by $70 \%$ evaporation in either a rotary evaporator (Quickfit) or Speed-vac freeze-dryer (Edwards). The extract was then treated with $30 \%$ absolute alcohol at $-15^{\circ} \mathrm{C}$. by gentle $\bar{\partial}$ mixing and allowed to act for one hour, when it was

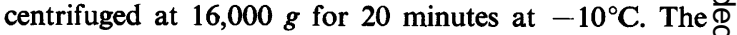
supernatant was decanted and treated with a further aliquot of alcohol to give a concentration of $60 \%$ alcohol. 음 This was again allowed to act for one hour and centrifuged as before. To this supernatant $90 \%$ acetone was added and left for one hour and centrifuged as before. It was? essential that the precipitants were kept at a low temp- $\overline{0}$ erature to avoid destruction of the colicine. Colicines A, $\mathrm{B}, \mathrm{D}, \mathrm{K}$, and S4 were precipitated by $60 \%$ alcohol, colicine $\mathrm{V}$ by $90 \%$ acetone.

\section{RESULTS}

THE PRODUCTION OF COLICINES ON AGAR The $D$ range of titres which were obtained in experiments

TABLE I

THE RANGE OF TITRES OBTAINED ON ROUTINE EXTRACTION FROM AGAR ${ }^{1}$

\begin{tabular}{|c|c|c|c|}
\hline Colicine & Range of Titre & $\begin{array}{l}\text { Number of } \\
\text { Assays }\end{array}$ & Mean Titre \\
\hline $\mathbf{A}$ & $4-256$ & 4 & 97 \\
\hline B & $16-128$ & 4 & 56 \\
\hline D & $2-256$ & 4 & 67 \\
\hline $\mathbf{K}$ & $1,280-8,192$ & 8 & 4,703 \\
\hline S4 & Neat-256 & 6 & 40 \\
\hline V & $2-1,024$ & 23 & 117 \\
\hline
\end{tabular}

${ }^{1}$ Titres are given as reciprocal dilutions. 
TABLE II

THE NUMBER OF UNITS OF EACH COLICINE REQUIRED TO INHIBIT EACH INDICATOR

Colicine Indicators

Maximum

\begin{tabular}{lllllllllllllllll}
\hline 2 & 56 & 17 & $2 M$ & 38 & $56 / 56$ & $56 / 98$ & $R I$ & $R 6$ & $M / 19$ & $2 / 7$ & $2 / 64$ & $2 / 15$ & $R / 5$ & Row & Phi & Tested (units)
\end{tabular}

\begin{tabular}{|c|c|c|c|c|c|c|c|c|c|c|c|c|c|c|c|c|c|}
\hline \multirow[t]{2}{*}{$\mathbf{A}$} & c 100 & 50 & 100 & - & - & 100 & 100 & 100 & 50 & - & 100 & 500 & 100 & - & 1 & & \\
\hline & s 100 & 20 & 400 & - & - & 20 & 20 & 100 & 10 & - & 20 & 10 & 100 & - & 1 & 1 & 8,000 \\
\hline \multirow[t]{2}{*}{ B } & 10 & 50 & 10 & - & 10 & 10 & 10 & 10 & 20 & - & 10 & 10 & 10 & 10 & 5 & & \\
\hline & 10 & 10 & 10 & - & 10 & 10 & 10 & 10 & 10 & - & 10 & 10 & 10 & 10 & 5 & 1 & 2,000 \\
\hline \multirow[t]{2}{*}{ D } & 50 & 50 & 50 & - & 50 & 50 & 50 & 50 & 100 & - & 100 & 50 & 100 & 100 & 5 & & \\
\hline & 50 & 50 & 10 & - & 10 & 10 & 10 & 50 & 10 & - & 50 & 10 & 10 & 10 & 5 & 1 & 1,000 \\
\hline \multirow[t]{2}{*}{$\mathbf{K}$} & 5 & - & 5 & - & 5 & - & - & 5 & - & - & 10 & 5 & 5 & 5 & 5 & & \\
\hline & 5 & - & 5 & - & 5 & - & - & 5 & - & - & - & 5 & 5 & 5 & 5 & 1 & 100,000 \\
\hline \multirow[t]{2}{*}{ S4 } & - & - & - & - & - & - & - & - & - & - & - & - & - & - & 5 & & \\
\hline & - & - & - & - & - & - & - & - & - & - & - & - & - & - & 5 & 1 & 1,000 \\
\hline \multirow[t]{2}{*}{ V } & 10 & 1 & 1 & 10 & 5 & - & - & 20 & 1 & - & 1 & 1 & 1 & 500 & 1 & & \\
\hline & 5 & 1 & 1 & 10 & 5 & - & - & 10 & 1 & - & - & 10 & 10 & 20 & 1 & 1 & 2,000 \\
\hline
\end{tabular}

- $=$ No inhibition noted at maximum concentration

c $=$ Colindale subcultures

$\mathrm{s} \quad=$ Stock subcultures

using digest nutrient agar is shown in Table $\mathbf{I}$. Although the same methods of preparation of nutrient agar and for extraction of the colicines were used, there was a variation in the potency of the extracts with all the colicinogenic strains. This variation was not correlated with the amounts of agar used, which varied from $20 \mathrm{ml}$. to $100 \mathrm{ml}$. per plate. Differences in titre were not observed when samples of the same batch of medium were inoculated with the same culture of the colicinogenic strain. Where different colicines were produced on identical media, using the same inoculum dosage, there was also variation in potency between the colicines. Only one strain, E. coli, CL 131, producing colicine $\mathrm{K}$, has been consistent in its high yield of colicine.
MINIMUM DOSAGE OF COLICINE REQUIRED TO INHIBIT THE INDICATORS Using the 'unit' of colicine as the final dilution to inhibit the growth of the indicator E. coli phi, 3,004 assays were performed. In Table II, the dosage of the inhibition level of each of the colicines for a specific indicator is demonstrated. Not only was the sensitivity of the row of indicators for a particular colicine variable, but each indicator showed a variation in its sensitivity to different colicines.

As to be expected from an antibiotic, the zones of inhibition decreased with the reduction in dosage of the colicine. This applied to all the colicines and to all the indicators. The gradients of diffusion against the indicator $E$. coli phi, shown in Fig. 2, where the points of $1,10,100$, and 1,000 units have been

Diffusion Gradients of Colicine against E coli. phi.

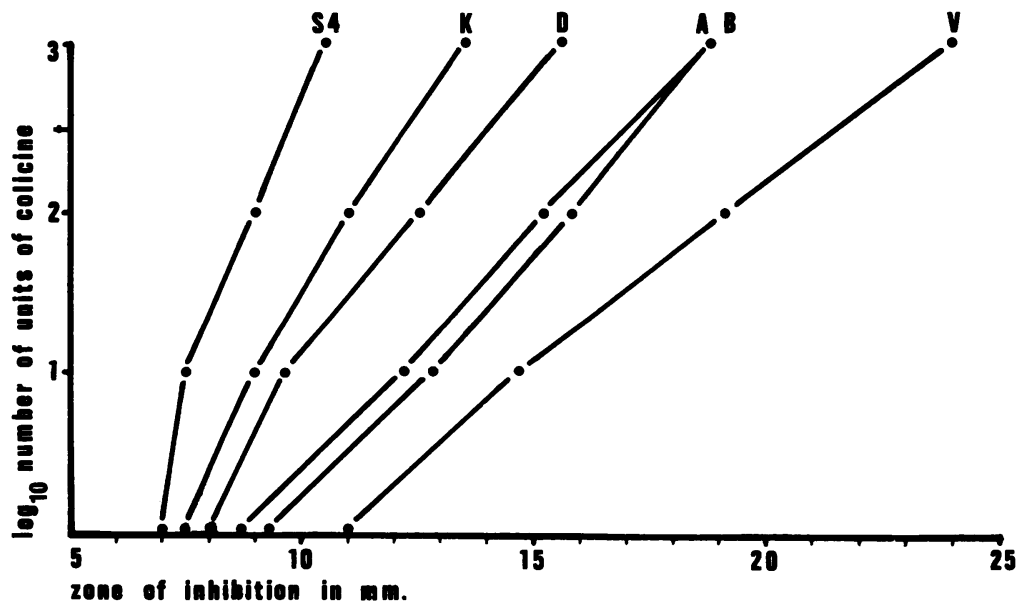

FIG. 2. The gradients have been made from between 21 and 43 assays of each colicine, in the range of 1 to 1,000 units. The indicator was $\mathrm{E}$. coli phi. 
TABLE III

PATTERN OF COLICINES AT THE RANGES OF TITRES NORMALLY PRODUCED Colicine Reciprocal of Titre Indicators

\begin{tabular}{|c|c|c|c|c|c|c|c|c|c|c|c|c|c|c|c|c|c|}
\hline \multirow[t]{2}{*}{ Colicine } & \multirow[t]{2}{*}{ Reciprocal of Titre } & \multicolumn{16}{|c|}{ Indicators } \\
\hline & & 2 & 56 & 17 & $2 M$ & 38 & $\frac{56}{56}$ & $\frac{56}{98}$ & $\boldsymbol{R} \boldsymbol{I}$ & $R 6$ & M19 & $\frac{2}{7}$ & $\frac{2}{64}$ & $\frac{2}{15}$ & $R 5$ & Row & Phi \\
\hline \multirow[t]{2}{*}{$\mathbf{A}$} & 4 & - & - & - & - & - & - & - & - & - & - & - & - & - & - & + & + \\
\hline & 256 & + & + & - & -1 & - & + & + & + & + & - & + & $t^{2}$ & + & - & + & + \\
\hline \multirow[t]{2}{*}{$\mathbf{B}$} & 16 & + & $t^{2}$ & + & - & + & + & + & + & +2 & - & + & + & + & + & + & + \\
\hline & 128 & + & + & + & - & + & + & + & + & + & - & + & + & + & + & + & + \\
\hline \multirow[t]{2}{*}{ D } & 2 & - & - & - & - & - & - & - & - & - & - & - & - & - & - & - & + \\
\hline & 256 & + & + & + & - & + & + & + & + & + & - & + & + & + & + & + & + \\
\hline \multirow[t]{2}{*}{$\mathbf{K}$} & 1,000 & + & - & + & - & + & - & - & + & - & - & -1 & + & + & + & + & + \\
\hline & 8,000 & + & - & + & - & + & - & - & + & - & - & -1 & + & + & + & + & + \\
\hline \multirow[t]{2}{*}{ S4 } & Neat & - & - & - & - & - & - & - & - & - & - & - & - & - & - & - & + \\
\hline & 256 & - & - & - & - & - & - & - & - & - & - & - & - & - & - & + & + \\
\hline \multirow[t]{2}{*}{ V } & & - & + & + & - & - & - & - & - & + & - & - & -1 & -1 & - & + & $t^{2}$ \\
\hline & 1,024 & + & + & + & + & + & - & - & + & + & - & - & + & + & + & + & $t^{1}$ \\
\hline
\end{tabular}

TABLE IV

THE COLICINE SENSITIVITY OF PARENT INDICATORS AND THEIR DERIVATIVES

Colicine

Indicators: Parent and Derivatives

\begin{tabular}{|c|c|c|c|c|c|c|c|c|c|}
\hline 2 & $2 / 15$ & $2 / 64$ & $R \mathbf{R}$ & $2 / 7$ & $R 5$ & 56 & $56 / 56$ & $56 / 98$ & $R 6$ \\
\hline
\end{tabular}

Inhibition Titre in Units

\begin{tabular}{|c|c|c|c|c|c|c|c|c|c|c|c|}
\hline \multirow[t]{2}{*}{$\mathbf{A}$} & c & 100 & 100 & 500 & 100 & 100 & - & 50 & 100 & 100 & 50 \\
\hline & $\mathbf{s}$ & 100 & 100 & 10 & 100 & 20 & - & 20 & 20 & 20 & 10 \\
\hline \multirow[t]{2}{*}{ B } & c & 10 & 10 & 10 & 10 & 10 & 10 & 50 & 10 & 10 & 20 \\
\hline & $\mathbf{s}$ & 10 & 10 & 10 & 10 & 10 & 10 & 10 & 10 & 10 & 10 \\
\hline \multirow[t]{2}{*}{$\mathbf{D}$} & c & 50 & 100 & 50 & 50 & 100 & 100 & 50 & 50 & 50 & 100 \\
\hline & $\mathbf{s}$ & 50 & 10 & 10 & 50 & 50 & 10 & 50 & 10 & 10 & 10 \\
\hline \multirow[t]{2}{*}{$\mathbf{K}$} & c & 5 & 5 & 5 & 5 & 10 & 5 & - & - & - & - \\
\hline & $\mathbf{s}$ & 5 & 5 & 5 & 5 & - & 5 & - & - & - & - \\
\hline \multirow[t]{2}{*}{ S4 } & c & - & - & - & - & - & - & - & - & - & - \\
\hline & $\mathbf{s}$ & - & - & - & - & - & - & - & - & - & - \\
\hline \multirow[t]{2}{*}{ V } & c & 10 & 1 & 1 & 20 & 1 & 500 & 1 & - & - & - \\
\hline & $\mathbf{s}$ & 5 & 10 & 10 & 10 & - & 20 & 1 & - & - & - \\
\hline
\end{tabular}

$\square$ Parent strain of subsequent indicators $\mathrm{c}=$ Colindale subculture

$\mathrm{s}=$ Stock subculture

plotted, have been constructed from between 21 and 43 assays, with a mean of 31 assays.

INHIBITORY PATTERN AND COLICINE TITRE From Tables II and III it is apparent that the pattern of a colicine is related to its titre and the susceptibility of the indicator strain. This has been noted particularly with colicine $A$ and colicine $D$, where the patterns obtained by the lower dosage of colicine extracts were markedly different from those obtained with the higher yield extracts. On the other hand, the patterns of colicines $B, K$, and $S_{4}$ showed no variation between the high and low dosage.

Another variation in patterns has been noted with the strain of indicator. In the main our stock strains, which have given the same patterns for some six years, had a similar sensitivity to the Colindale strains. Nevertheless there were some import- ant exceptions; for example, the indicator $2 / 7$, which was resistant to colicine $\mathbf{K}$ with our strain, $₹$ was sensitive with the Colindale subcultures. Witho colicine $\mathrm{V}$ there was a similar variation. Conversely, our stock indicator 2/64 was sensitive to colicine $A$ O at the upper level of dosage whereas at this level the Colindale strain was resistant.

A further aspect of this feature is shown in Tablen IV where the derivatives of the indicator strains were compared with the parent strains for sensitivityo to the different colicines.

DISCUSSION

When Fredericq (1948) described the development of resistant variants of an indicator strain to $\mathrm{a} \stackrel{\mathrm{P}}{\mathrm{P}}$ colicine, a practicable method of colicine typing 
became available. A further application of typing was described by Abbott and Shannon (1958), who used a range of Shigella indicators to give patterns of sensitivity and resistance of colicinogenic Shigella sonnei. Several of their Shigella indicators were mutants obtained from 'wild' strains of Shigella sonnei (Abbott and Shannon, 1958; Hart, 1965). A difficulty in using mutants is the maintenance of the stability of the variable factor, in this case colicine sensitivity, and although we have maintained the sensitivity of our stock strains for six years, these indicator strains are different in sensitivity to those used at the Dysentery Reference Laboratories, Colindale. This has been shown by comparing the sensitivity of the two sets of indicators to the six colicines used in this study. Since the patterns produced by our stock strains of indicators has remained unchanged for six years, as far as purified colicines $\mathrm{V}$ and $\mathrm{K}$ are concerned, the alteration in sensitivity must have occurred in our original sample and has remained stable since. The main difference in sensitivity has occurred with the indicator strain 2/7: our stock strain was resistant to the action of both colicine $\mathrm{V}$ and $\mathrm{K}$, even in high titre, whereas the Colindale strain was sensitive to relatively low dosage of both of these colicines. When one considers the parentage of the indicators (Table IV), the differences between the two sets of indicators is shown to be most marked with the derivatives rather than the parents.

Another variable in typing by colicine production has been the potency of the colicine found in the extracts. This feature has been noted before when different media have been compared(McGeachie and McCormick, 1963; Papavassiliou, 1963) but even where the same medium has been used we have found wide variations in the amounts of colicine present in the extracts, which have occurred despite the standardization of technique and media. The importance of this is emphasized when one compares the patterns produced by the two levels of potency present in the routine extracts (Table III). With our stock strains of indicators, no alteration in pattern was noted with the two potencies of colicines $\mathrm{B}, \mathrm{K}$, and $\mathrm{S}_{4}$; however, colicine $B$ did show a change in the inhibition of the Colindale strains of indicators 56 and R6. Clear differences in patterns were shown with the colicines A, D and, to a lesser extent, V, which would lead to discrepancies in results dependent on the potency. Although the potency may not be a critical factor in a strain which is a good producer of colicine, for example, $E$. coli $\mathrm{Cl} 131$ producing colicine $\mathrm{K}$, one cannot confirm this without testing the titre. The zone of diffusion from a stroke is no criterion since this depends on the molecular size of the colicine and, as we have shown with our gradients of diffusion, the same potency in two colicines can vary widely in the zone of inhibition.

We have $E$. coli phi as our indicator for potency since the majority of our routine test strains are Escherichia coli and we have found that this indicator is the most sensitive. However, for other species, for example, Shigella sonnei, $E$. coli phi may be insensitive to some of the bacteriocins and an additional indicator; for Shigellae sonnei we include Shigella sonnei strain 17, which may be required to avoid missing a colicinogenic strain. Indeed with Shigella sonnei, we have noted that some strains which were untypable by the standard stroke method (Abbott and Shannon, 1958; Gillies, 1964) have produced recognized patterns when concentrated tenfold (unpublished results).

In view of our investigations we feel that for the routine use of colicine production as a typing marker, two steps are essential. First the titre of the colicine used for the production of a pattern should be known. One may then use the extract at this concentration and compare the patterns produced by a similar concentration of known colicines, or one may adjust the titre, either by concentration or by dilution, to a suitable level of, say, 100 units. Secondly, the indicators should be examined at frequent intervals using known concentrations of colicines in order to avoid a change in the sensitivity. We feel that by using these quantitative techniques the method of colicine typing will become more accurate and consistent.

We wish to express our thanks to the following colleagues for strains of organisms used in this study: Professor P. Fredericq, Dr. R. R. Gillies, Dr. W. F. Goebel, Dr. B. A. D. Stocker, and Mrs. Judith Goodban. We acknowledge our appreciation to Scottish Hospital Endowments Research Trust for assistance with Grant HERT 215. We are grateful to Mrs. Catherine Graham, A.I.M.L.T., for technical assistance and to Mr. T. Parker for the photographs.

\section{REFERENCES}

Abbott, J. D., and Shannon, R. (1958). J. clin. Path., 11, 71.

Cradock-Watson, J. E. (1965). Zbl. Bakt., I. Abt. Orig., 196, 385.

Cruickshank, R. (1960). Mackie and McCartney's Handbook of Bacteriology, 10th ed., Livingstone, Edinburgh.

Darrell, J. H., and Wahba, A. H. (1964). J. clin. Path., 17, 236. Fredericq, P. (1948). Rev. belge Path., 19, suppl. 4.

, Thibault, J., and Gratia, A. (1946). C. R. Soc. Biol. (Paris), $140,1035$.

Gillies, R. R. (1964). J. Hyg.(Lond.), 62, 1.

-, and Govan, J. R. W. (1966). J. Path. Bact., 91, 339.

Goebel, W. F., Barry, G. T., and Shedlovsky, T. (1956). J. exp. Med., 103, 577.

Hamon, Y. (1959). Ann. Inst. Pasteur, 96, 614.

Hart, J. M. (1965). Zbl. Bakt., I. Abt. Orig., 196, 365.

Linton, K. B. (1960). J. clin. Path., 13, 168.

McGeachie, J. (1965). Zbl. Bakt., I Abt. Orig., 196, 377.

- , and McCormick, W. (1963). J. clin. Path., 16, 278.

Osman, M. A. M. (1965). Ibid., 18, 200.

Papavassiliou, J. (1963). Path. et Microbiol. (Basel), 26, 74.

Shannon, R. (1957). J. med. Lab. Technol., 14, 199.

Wahba, A. H. (1965). Zbl. Bakt., I. Abt. Orig., 196, 389. 


\section{Letter to the Editor}

\section{A CAUSE OF ERROR IN THE DETERMINATION OF THE HAEMATOCRIT USING THE SMA -4}

Sir,

One of the advances in automation of haematology has been the introduction of the AutoAnalyzer SMA.4. From a single sample of blood this instrument determines four parameters, viz., the haematocrit, the haemoglobin, the red cell and the white cell counts. In conjunction with a data logger, the MCH, MCHC, and MCV may be calculated. In our experience the results of the haematocrit determinations have not always been reliable.

In the SMA-4 the electrical conductivity of a sample is taken as an index of its haematocrit The conductivity is a function of the concentration of ions present. Therefore, an excess of ions supplied by the anticoagulant EDTA (dipotassium salt) could cause inaccuracy. While comparing the results of packed cell volumes done on the SMA-4 and by centrifugation in the microhaematocrit method, some discrepancies were apparent. These occurred when the anticoagulated bottles were not properly filled.

It has already been demonstrated by Pennock and Jones $^{1}$ that excess concentration of EDTA reduces the

\section{A simple device for microdialysis - concluded}

facilitates the decision as to which of possibly a large number of fractions are worth pooling and concentrating in bulk. The method has been found to be useful in the analysis of column-separated fractions of antibodies and antigens, as well as eluates from red cells, which had previously adsorbed a variety of immunologically active proteins. Additionally, the device is suitable for dialysing small volumes of protein solution to change ionic condition, or to remove low molecular weight substances such as 2-mercaptoethanol from sera treated with this agent. packed cell volumes when determined by the microhaematocrit method. In order to investigate the effect of excess EDTA on the conductivity method for packed cell volumes, a series of experiments was set up. Blood from several normal individuals was added to eight anticoagulated tubes to give a final concentration ranging from $2 \mathrm{mg}$ to $9 \mathrm{mg}$ of EDTA per millilitre of blood. The results are set out in the table below:

$\begin{array}{lrrrrrrrr}\text { Concentration of EDTA (mg/ml blood) } & 2 & 3 & 4 & 5 & 6 & 7 & 8 & 9 \\ \text { Mean \% error of SMA-4 method } & 0 & 1 & 4 & 8 & 11 & 13 & 18 & 21 \\ \begin{array}{l}\text { Mean \% error of microhaematocrit } \\ \text { method }\end{array} & 0 & 0 & 0 & 1 & 1 & 1 & 2 & 2\end{array}$

In the microhaematocrit method the concentration of EDTA in this range does not alter the haematocrit reading significantly. This finding is in accordance with that of Pennock and Jones ${ }^{1}$. When samples of blood with high concentration of EDTA are used, the haematocrit determination, carried out by a conductance method, is significantly altered.

To produce a final concentration of $2 \mathrm{mg} / \mathrm{ml}$ of blood as recommended by Dacie and Lewis ${ }^{2}$, we add $10 \mathrm{mg}$ of EDTA to each of our haematology sample bottles.

If less than $4 \mathrm{ml}$ of blood is added to the sample bottle the resultant concentration of anticoagulant is such as to produce a significant error in the haematocrit reading. Therefore, if the SMA-4 is to be used for haematocrit determinations, it is important that the blood should be mixed with the anticoagulant in the correct proportions.

In the determination of the other parameters on the instrument, the concentration of anticoagulant is less important.

T. J. R. LAPPIN AND A. LAMONT Department of Clinical Pathology, Royal Victoria Hospital, Belfast 12

\section{REFERENCES}

${ }^{1}$ Pennock and Jones, J. clin. Path., 1966, 19.

${ }^{2}$ Dacie, J. V., and Lewis, S. M. (1963). Practical Haematology, 3rd ed. p.4. Churchill, London.

\section{CORRECTION}

We print below the corrected Table III of the paper by J. McGeachie and W. McCormick 'Importance of potency in typing by colicine production' (J. clin. Path., 20, 887-891).

TABLE III

PATTERN OF COLICINES AT THE RANGES OF TITRES NORMALLY PRODUCED

Colicine Reciprocal of Titre Indicators

\begin{tabular}{|c|c|c|c|c|c|c|c|c|c|c|c|c|c|c|c|}
\hline 2 & 56 & 17 & $2 M$ & 38 & $\frac{56}{56}$ & $\frac{56}{98}$ & $R \boldsymbol{R}$ & $R 6$ & M19 & $\begin{array}{l}2 \\
\overline{7}\end{array}$ & $\frac{2}{64}$ & $\frac{2}{15}$ & $R 5$ & Row & Phi \\
\hline-- & - & - & - & - & - & - & - & - & - & - & - & - & - & + & + \\
\hline+ & + & -1 & - & - & + & + & + & + & - & + & $+^{2}$ & + & - & + & + \\
\hline+ & $t^{2}$ & + & - & + & + & + & + & $t^{2}$ & - & + & + & + & + & + & + \\
\hline+ & + & + & - & + & + & + & + & + & - & + & + & + & + & + & + \\
\hline- & - & - & - & - & - & - & - & - & - & - & - & - & - & - & + \\
\hline+ & + & + & - & + & + & + & + & + & - & + & + & + & + & + & + \\
\hline+ & - & + & - & + & - & - & + & - & - & -1 & + & + & + & + & + \\
\hline+ & - & + & - & + & - & - & + & - & - & -1 & + & + & + & + & + \\
\hline- & - & - & - & - & - & - & - & - & - & - & - & - & - & - & + \\
\hline- & - & - & - & - & - & - & - & - & - & - & - & - & - & + & + \\
\hline- & + & + & - & - & - & - & - & + & - & -1 & -1 & -1 & - & + & + \\
\hline+ & + & + & + & + & - & - & + & + & - & -1 & + & + & + & + & + \\
\hline
\end{tabular}

$+=$ inhibition of indicator

$-=$ no inhibition of indicator

1inhibition at this level with Colindale subculture

${ }^{2}$ no inhibition at this level with Colindale subculture 